\title{
Supersymmetry and Inflation
}

\author{
G. Lazarides \\ Physics Division, School of Technology, \\ University of Thessaloniki, Thessaloniki 540 06, Greece
}

\begin{abstract}
A variant of hybrid inflation which is applicable in a wide class of supersymmetric grand unified models and reproduces the observed temperature perturbations of cosmic background radiation with natural values of the parameters is presented. The theory is consistent with the unification of the minimal supersymmetric standard model gauge couplings as measured at LEP. The termination of inflation is smooth and does not produce any topological defects. Numerical investigation of the cosmological evolution of the system shows that for almost all initial values of the fields we do get an adequate amount of inflation. Finally, the "reheating" process following inflation and the production of the baryon asymmetry of the universe via a primordial lepton asymmetry are briefly discussed and some important implications for right handed neutrino Majorana masses are investigated.
\end{abstract}

Some years ago Linde [1] has proposed, in the context of non-supersymmetric grand unified theories (GUTs), a clever infationary scenario which he called hybrid inflation. The idea was to use two real scalar fields $\chi$ and $\sigma$ instead of one that is normally used. The field $\chi$ provides the vacuum energy which drives inflation while $\sigma$ is the slowly varying field during inflation. The main advantage of this scenario is that it can reproduce the observed temperature fluctuations of cosmic background radiation (CBR) with "natural" values of the parameters of the theory in contrast to previous realizations of inflation which require extremely small coupling constants. The potential utilized by Linde is

$$
V(\chi, \sigma)=\kappa^{2}\left(\mu^{2}-\frac{\chi^{2}}{4}\right)^{2}+\frac{\lambda^{2} \chi^{2} \sigma^{2}}{4}+\frac{m^{2} \sigma^{2}}{2}
$$

where $\kappa, \lambda$ dimensionless positive coupling constants and $\mu, \mathrm{m}$ mass parameters. The vacua lie at $\langle\chi\rangle= \pm 2 \mu,\langle\sigma\rangle=0$. Putting $\mathrm{m}=0$ for the moment, we observe that the potential possesses an exact flat direction at $\chi=0$ with $V(\chi=0, \sigma)=\kappa^{2} \mu^{4}$. The mass squared of the field $\chi$ along this flat direction is given by $m_{\chi}^{2}=-\kappa^{2} \mu^{2}+\frac{1}{2} \lambda^{2} \sigma^{2}$ and remains non-negative for $\sigma \geq \sigma_{c}=\sqrt{2} \kappa \mu / \lambda$. This means that, at $\chi=0$ and $\sigma \geq \sigma_{c}$, we obtain a valley of minima with flat bottom. Reintroducing the mass parameter $\mathrm{m}$ in eq.(1) we observe that this valley acquires a non-zero slope. A region of the universe where $\chi$ and $\sigma$ happen to be almost uniform with negligible kinetic energies and with values close to the bottom of the valley of minima follows this valley in its subsequent evolution and undergoes inflation. The temperature fluctuations of CBR produced during this inflation can be estimated to be

$$
\frac{\delta T}{T} \simeq\left(\frac{32 \pi}{45}\right)^{1 / 2} \frac{V^{3 / 2}}{M_{P}^{3} V^{\prime}} \simeq\left(\frac{16 \pi}{45}\right)^{1 / 2} \frac{\lambda \kappa^{2} \mu^{5}}{M_{P}^{3} m^{2}}
$$

where $M_{P}=1.22 \times 10^{19} \mathrm{GeV}$ is the Planck mass, prime denotes derivative with respect to $\sigma$ and $V, V^{\prime}$ are evaluated at $\chi=0$. The Cosmic Background Explorer (COBE) result, $\delta T / T \simeq 6.6 \times 10^{-6}$, can 
then be reproduced with $\mu=2.86 \times 10^{16} \mathrm{GeV}$ [the supersymmetric (SUSY) GUT vacuum expectation value $($ vev) $]$ and $m \simeq \kappa \sqrt{\lambda} 1.3 \times 10^{15} \mathrm{GeV} \sim 10^{12} \mathrm{GeV}$ for $\kappa, \lambda \sim 10^{-2}$. Inflation terminates abruptly at $\sigma=\sigma_{c}$ and is followed by a "waterfall", i.e., sudden entrance into an oscillatory phase about a global minimum. Since the system can fall into either of the two available global minima with equal probability, topological defects can be easily produced if they are predicted by the particular particle physics model one is considering.

The hybrid inflationary scenario is "tailor made" for application to SUSY GUTs except that the mass of $\sigma, m$, is unacceptably large for SUSY where all scalar fields acquire masses of order $m_{S} \sim 1$ TeV from SUSY breaking. To see this consider a SUSY GUT with a (semi-simple) gauge group $G$ of rank $\geq 5:$

$$
G \rightarrow G_{S} \equiv S U(3)_{c} \times S U(2)_{L} \times U(1)_{Y}
$$

at a scale $M_{X} \simeq 2 \times 10^{16} \mathrm{GeV}$. The spectrum of the theory below $M_{X}$ is assumed to coincide with the minimal supersymmetric standard model (MSSM) spectrum plus standard model (SM) singlets so that the successful predictions for $a_{s}, \sin ^{2} \theta_{w}$ are retained. The theory may also possess global symmetries. The breaking in eq.(3) is achieved through the superpotential

$$
W=\kappa s\left(-\mu^{2}+\bar{\phi} \phi\right)
$$

where $\bar{\phi}, \phi$ is a conjugate pair of SM singlet left handed superfields which belong to non-trivial representations of $G$ and reduce its rank by their vevs and $s$ is a gauge singlet left handed superfield. This superpotential is the most general renormalizable superpotential consistent with a U(1) R-symmetry under which $W \rightarrow e^{i \theta} W, s \rightarrow e^{i \theta} s, \bar{\phi} \phi \rightarrow \bar{\phi} \phi$ and gives the potential

$$
\begin{gathered}
V=\kappa^{2}\left|\mu^{2}-\bar{\phi} \phi\right|^{2}+\kappa^{2}|s|^{2}\left(|\phi|^{2}+|\bar{\phi}|^{2}\right) \\
+D-\text { terms. }
\end{gathered}
$$

Restricting ourselves to the D-flat direction $\bar{\phi}^{*}=\phi$ which contains the SUSY minima and performing appropriate gauge and $R$-transformations we can bring $s, \bar{\phi}, \phi$ on the real axis, i.e., $s \equiv \sigma / \sqrt{2}, \bar{\phi}=$ $\phi \equiv \chi / 2$, where $\sigma, \chi$ are normalized real scalar fields. The potential then takes the form in eq.(1) with $\kappa=\lambda$ and $m=0$ and, thus, Linde's potential for hybrid inflation is almost obtainable from SUSY GUTs but without the mass term of $\sigma$ which is,however, crucial since it provides the slope of the valley of minima necessary for inflation.

One way to obtain a valley of minima useful for inflation is to replace the renormalizable trilinear term in $W$ in eq.(4) by the next order non- renormalizable coupling:

$$
W=s\left(-\mu^{2}+\frac{(\bar{\phi} \phi)^{2}}{M^{2}}\right)
$$

where $M \sim 10^{18} \mathrm{GeV}$ and is related to the scale which controls the non- renormalizable contributions (The coupling constant $\kappa$ is absorded in $\mu$ and M) [2]. This is achieved by imposing an extra $Z_{2}$ discrete symmetry under which $\bar{\phi} \phi \rightarrow-\bar{\phi} \phi$. Indeed $W$ in eq.(6) contains all the dominant terms consistent with this discrete, the $R$ - and the gauge symmetry. The potential obtained for $W$ in eq.(6) is then

$$
V(\chi, \sigma)=\left(\mu^{2}-\frac{\chi^{4}}{16 M^{2}}\right)^{2}+\frac{\chi^{6} \sigma^{2}}{16 M^{4}}
$$

with SUSY vacua at $\left.\langle\chi\rangle= \pm 2(\mu M)^{1 / 2},<\sigma\right\rangle=0$. The SUSY GUT scale is $M_{X}=g(\mu M)^{1 / 2}$ with $g \simeq 0.7$ being the unified gauge coupling constant. The potential in eq.(7) although of quite 
similar form with the potential in eq.(1) with $m=0$ possesses some crucial differences. The flat direction at $\chi=0$ with $V(\chi=0, \sigma)=\mu^{4}$ is now a local maximum in the $\chi$ direction for all values of $\sigma$ but two valleys of local minima develop on both sides and close to this flat direction at $\chi^{2} \simeq$ $4 \mu^{2} M^{2} / 3 \sigma^{2}\left(\sigma^{2}>>\mu M\right)$ with $V \simeq \mu^{4}\left(1-2 \mu^{2} M^{2} / 27 \sigma^{4}\right)$. These valleys have an inbuilt slope and thus they can, in principle, be used for inflation. Indeed, as one can easily show, a region of the universe with $\sigma, \chi$ field values close to the bottom of one of these valleys follows this valley in its subsequent evolution and inflates till $\sigma \simeq \sigma_{o} \simeq\left(2 M_{P} / 9 \sqrt{\pi}(\mu M)^{1 / 2}\right)^{1 / 3}(\mu M)^{1 / 2}$. The number of e-foldings produced when the $\sigma$ slowly varies from an initial value $\sigma$ till $\sigma_{o}$ is estimated to be $N(\sigma) \simeq\left(3 \sqrt{2 \pi} / 2 \mu M M_{P}\right)^{2} \sigma^{6}$ and, consequently, the value of $\sigma$ at which our present horizon size crossed outside the inflationary horizon is $\sigma_{H}=\left(9 N_{H} / 2\right)^{1 / 6} \sigma_{o}$, where $N_{H} \simeq 60$ is the number of e-foldings suffered by our present horizon during inflation. After the end of inflation at $\sigma_{o}$, the fields $\sigma, \chi$ enter smoothly into an oscillatory phase about the SUSY vacuum with frequencies $m_{\sigma}=m_{\chi}=2 \sqrt{2}(\mu / M)^{1 / 2} \mu$. This is the reason for calling our scenario smooth hybrid inflation.

Now, calculating the scalar part of $\delta T / T$ we find

$$
\left(\frac{\delta T}{T}\right)_{S} \approx \frac{1}{\sqrt{5}}\left(\frac{6}{\pi}\right)^{1 / 3} N_{H}^{5 / 6}\left(\frac{M_{X}}{g}\right)^{10 / 3} M_{P}^{-4 / 3} M^{-2}
$$

and the COBE result can be reproduced with $M \approx 8.6 \times 10^{17} \mathrm{GeV}, \mu \approx 9.5 \times 10^{14} \mathrm{GeV}$.Consequently, the observed temperature fluctuations of CBR can be obtained with "natural" values of the parameters (in particular, $M \sim 10^{18} \mathrm{GeV}$ ) and consistently with the SUSY GUT scale $M_{X} \approx 2 \times 10^{16} \mathrm{GeV}$. Some comments are in order:(1) The gravitational wave part of $\delta T / T,(\delta T / T)_{T} \sim 10^{-9}<<(\delta T / T)_{S}$ and the spectral index $n \approx 0.97$. (2) Since the system follows a particular valley of minima from the beginning it ends up at a particular SUSY vacuum and, thus, no topological defects are produced.(3) Replacement of global SUSY with supergravity (SUGRA) makes inflation in general impossible since it produces a mass for the inflaton higher than the Hubble constant during inflation. In our model, due to the R-symmetry, this does not happen so we can hope that SUGRA may not destroy the whole picture. (4) The relevant part of inflation takes place between the $\sigma$ field values $\sigma_{H} \approx 2.7 \times 10^{17} \mathrm{GeV}$ and $\sigma_{o} \approx 1.1 \times 10^{17} \mathrm{GeV}$ which are much smaller than $M_{c} \equiv M_{P} / \sqrt{8 \pi} \simeq 2.4 \times 10^{18} \mathrm{GeV}$ and, thus, SUGRA corrections are under control.

An inflationary scenario is fully successful if it is obtainable for a wide class of "natural" initial conditions, i.e., initial values of the fields and their time derivatives. We, thus, tried to specify initial values of $\chi, \sigma$ (for simplicity we put their time derivatives equal to zero) for which the system falls at the bottom of the valley of minima at a $\sigma \geq \sigma_{H}$ so that its subsequent evolution along the valley produces adequate amount of inflation [3]. The evolution equations are

$$
\begin{gathered}
\ddot{\chi}+3 H \dot{\chi}-\frac{\chi^{3}}{2 M^{2}}\left(\mu^{2}-\frac{\chi^{4}}{16 M^{2}}\right)+\frac{3 \chi^{5} \sigma^{2}}{8 M^{4}}=0, \\
\ddot{\sigma}+3 H \dot{\sigma}+\frac{\chi^{6} \sigma}{8 M^{4}}=0,
\end{gathered}
$$

where

$$
H=\left(\frac{8 \pi}{3}\right)^{1 / 2} M_{P}^{-1}\left(\frac{1}{2} \dot{\chi}^{2}+\frac{1}{2} \dot{\sigma}^{2}+V(\sigma, \chi)\right)^{1 / 2}
$$

is the Hubble constant and overdots denote time derivatives. We integrated these equations numerically for initial values of the fields $0.1 \leq \hat{\sigma} \equiv \sigma / M_{P} \leq 1.2,0.01 \leq \hat{\chi} \equiv \chi / M_{P} \leq 0.5$ and the results are shown in Figure 1. Each point on the $\hat{\sigma}-\hat{\chi}$ plane corresponds to a given set of initial conditions and 
depicts a definite evolution pattern of the system. Filled circles correspond to an evolution pattern where both $\chi$ and $\sigma$ oscillate and fall into a SUSY vacuum without producing any appreciable amount of inflation. Open triangles cover a region where $\sigma>>$ initially and depict a pattern where $\sigma$ decreases slowly and monotonically towards a constant value greater than $\sigma_{H}$ while $\chi$ oscillates and relaxes at the bottom of the valley. The subsequent evolution along the valley produces adequate amount of inflation. The limiting case $\sigma>>M_{P}>>\chi$ which lies deeply into the region of open triangles can be studied analytically and one finds very little variation of $\sigma$ in this case. Although the region of open triangles leads to adequate inflation we do not consider it as "natural" because it requires a considerable descrepancy between the values of $\chi$ and $\sigma$. Open circles which cover most of the diagram correspond to the following situation: Both fields start oscillating from the beginning but, due to energy transfer from $\chi$ to $\sigma$, the amplitude of $\sigma$ increases gradually and eventually the system gets trapped to an evolution pattern of the open triangle type leading again to adequate inflation. This interesting energy transfer phenomenon between the two coupled oscillating fields is very important because it ensures adequate inflation in the most "natural" region of initial conditions $\left(\hat{\chi} \sim \hat{\sigma} \sim 10^{-1}\right)$ with "natural" initial energy density values. To avoid strong influence from SUGRA we also considered initial field values $0.01 \leq \hat{\sigma}, \hat{\chi} \leq 0.1$. We found that almost all points are of the open circle type. The overall conclusion is that almost all initial conditions give adequate smooth hybrid inflation. This fact together with the other advantages of this scenario makes it very "natural" and successful.

Finally we will discuss the "reheating" process [4]. After the end of inflation the inflaton, which consists of the two complex scalar fields $s$ and $\theta=(\delta \phi+\delta \bar{\phi}) / \sqrt{2}$, where $\delta \phi=\phi-(\mu M)^{1 / 2}, \delta \bar{\phi}=$ $\bar{\phi}-(\mu M)^{1 / 2}$, with mass $m_{\text {inf }}=m_{s}=m_{\theta}=2 \sqrt{2}(\mu / M)^{1 / 2} \mu \approx 8.93 \times 10^{13} \mathrm{GeV}$, performs damped oscillations about the SUSY vacuum and eventually decays to light particles. We will assume that $\mathrm{s}$ decays faster and, thus, we will concentrate on the decay of $\theta$. Its dominant decay mode is in a pair of right handed neutrinos, i.e., $\theta \rightarrow \nu^{c} \nu^{c}$ through the superpotential term $\delta W=\left(M_{\nu^{c}} / 2 \mu M\right) \bar{\phi} \bar{\phi} \nu^{c} \nu^{c}\left(M_{\nu^{c}}\right.$ is the mass of the relevant right handed neutrino). The "reheat" temperature is estimated to be $T_{r} \approx(1 / 7)\left(\Gamma_{\theta} M_{P}\right)^{1 / 2} \approx 3.3 \times 10^{-2} M_{\nu^{c}}$, where $\Gamma_{\theta}$ is the decay rate of $\theta$. For simplicity, we will ignore the first family of quarks and leptons. The Majorana mass matrix of right handed neutrinos can be brought to the diagonal form $m=\operatorname{diag}\left(M_{1}, M_{2}\right)$ with $M_{1} \geq M_{2}>0$. The approximate see-saw light neutrino mass matrix $m_{D} \frac{1}{m} m_{D}\left(m_{D}\right.$ is the neutrino Dirac mass matrix) can then also be diagonalized by rotating to an appropriate basis of left handed neutrinos. In this basis of right and left handed neutrinos the elements of the Dirac mass matrix

$$
m_{D}=\left[\begin{array}{ll}
a & b \\
c & d
\end{array}\right]
$$

are not all independent. They can be expressed in terms of three complex parameters $a, d$ and $\eta: b=-\left(M_{2} / M_{1}\right)^{1 / 2} \eta a, c=\left(M_{1} / M_{2}\right)^{1 / 2} \eta d$ and the light neutrino masses take the form $m_{1}=|a|^{2}$ $\left|1+\eta^{2}\right| / M_{1}, m_{2}=|d|^{2}\left|1+\eta^{2}\right| / M_{2}$. Restricting ourselves to the case $|\eta| \sim 1$ and $M_{1} / M_{2}>>1$, we have $|a|>>|b|,|c|>>|d|$. Taking further $|c|<<|a|, a$ becomes the dominant element of $m_{D}$. Diagonalization of $m_{D}^{+} m_{D}$ under these conditions gives the approximate Dirac mass eigenvalues $|a|,|d|\left|1+\eta^{2}\right|$. Assuming that the dominant contributions to $m_{D}$ and the up type quark mass matrix coincide asymptotically we can then obtain the asymptotic relation $m_{t} \approx|a|$. We are now ready to draw some important conclusions. Suppose that the inflaton decays predominantly to the heaviest right handed neutrino with mass $M_{1}$. The well known gravitino problem requires $T_{r} \leq 10^{9} \mathrm{GeV}$ which in this case gives $M_{1} \leq 3 \times 10^{10} \mathrm{GeV}$ with the consequence that $m_{1} \approx m_{t}^{2}\left|1+\eta^{2}\right| / M_{1}>>100$ 
$\mathrm{eV}$. This is cosmologically unacceptable and thus we are led to the conclusion that the inflaton should decay to the second heaviest right handed neutrino. This requires that $M_{1} \geq m_{\text {inf }} / 2 \approx 4.47 \times 10^{13}$ $\mathrm{GeV}$ and $M_{2} \leq 3 \times 10^{10} \mathrm{GeV}$. The observed baryon asymmetry of the universe can be generated by first producing a primordial lepton asymmetry via the out-of-equilibrium decay of the right handed neutrinos which emerge from the decay of the inflaton. Non-perturbative effects at the electroweak transition convert a fraction of this asymmetry to the observed baryon asymmetry. The primordial lepton asymmetry is given by

$$
\frac{n_{L}}{s} \approx \frac{9}{8 \pi} \frac{T_{r}}{m_{\text {inf }}} \frac{M_{2}}{M_{1}} \frac{m_{t}^{2}}{v^{2}} \operatorname{Im}\left(\eta^{* 2} /|\eta|^{2}\right)
$$

( $v=174 \mathrm{GeV}$ is the electroweak scale and $m_{t} \approx 110 \mathrm{GeV}$ asymptotically) and is maximized by saturating the previously obtained bounds on $M_{1}, M_{2}$. Infact $M_{1} \approx 4.47 \times 10^{13} \mathrm{GeV}$ and $M_{2} \approx 3 \times 10^{10}$ $\mathrm{GeV}$ give $n_{L} / s \leq 10^{-9}$ which is satisfactory. We see that the requirement of a successful "reheating" process specifies the Majorana masses of the two heaviest right handed neutrinos with considerable accuracy.

\section{References}

[1] A. D.Linde, Phys. Rev. D49(1994)748

[2] G. Lazarides and C. Panagiotakopoulos, Phys. Rev. D52(1995) R559

[3] G. Lazarides, C. Panagiotakopoulos and N.D. Vlachos, Phys. Rev. D54(1996)54

[4] G. Lazarides, R. K. Schaefer and Q. Shafi (in preparation)

Figure 1. Evolution patterns for the $\hat{\sigma}-\hat{\chi}$ system. 


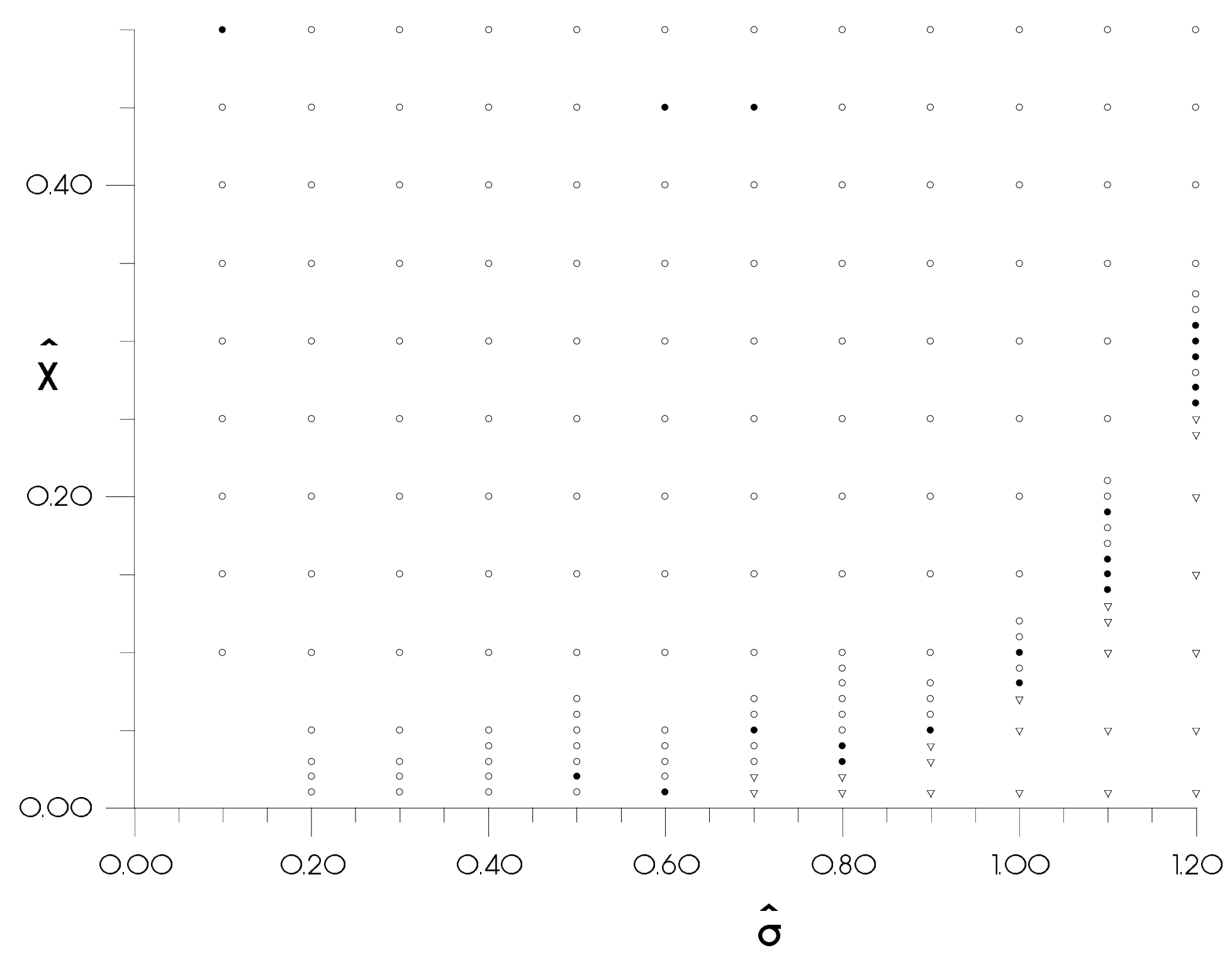

Flg. 1 